\title{
Bipolar disorders in the Arab world: a critical review
}

\author{
Ziad Kronfol, ${ }^{1,2,3}$ Mostafa Zakaria Khalil, ${ }^{2}$ Pankaj Kumar, ${ }^{1}$ Karsten Suhre, ${ }^{1}$ Elie Karam, ${ }^{4}$ \\ and Melvin Mclnnis ${ }^{3}$ \\ ${ }^{1}$ Department of Medical Education, Weill Cornell Medical College in Qatar, Doha, Qatar. ${ }^{2}$ Department of Psychiatry, Hamad \\ Medical Corporation, Doha, Qatar. ${ }^{3}$ Department of Psychiatry, University of Michigan Medical Center, Ann Arbor, Michigan. \\ ${ }^{4}$ Department of Psychiatry, Faculty of Medicine and Medical Sciences, University of Balamand, Beirut, Lebanon
}

Address for correspondence: Ziad Kronfol, M.D., Weill Cornell Medical College in Qatar, 445 E. 69th Street, Suite 432, Olin Hall, New York, NY 10021. zik2002@qatar-med.cornell.edu

Bipolar disorders are common psychiatric disorders that affect $1-5 \%$ of the population worldwide. Major advances in the epidemiology, pathophysiology, and treatment of the disorders have recently occurred. The majority of published reports, however, originate from the Western hemisphere, mostly Europe and the United States. There is a shortage of data from the Arab world on bipolar disorders. In an era of globalization and rapid communication, it is not clear to what extent research findings pertaining to one part of the world are by necessity applicable to other parts. Psychiatric disorders are known to be affected by the culture in which they occur, and knowledge of variations in illness presentation in different ethnic groups is also increasing. However, knowledge of variations affecting Arab populations remains quite limited. This paper provides a critical review of the literature on bipolar affective disorders in the Arab world, pointing to major gaps in knowledge and future opportunities to fill these gaps.

Keywords: bipolar disorder; Arab world; Qatar; Lebanon; review

\section{Introduction}

The Arab world is a loose association of countries in the Middle East and North Africa that are connected by history, language, and culture. The total population exceeds 300 million people. The majority of Arabs are Muslim, although there are also large Muslim populations outside the Arab world and there is a considerable number of Arabs who are non-Muslim (e.g., Christian and Jewish). Wide discrepancies exist in the economies of countries such as the United Arab Emirates and Qatar, boasting per capita incomes among the highest in the world, and countries such as Djibouti and Somalia, which have some of the poorest. Biomedical research, particularly in the field of mental health, is relatively weak, often stigmatized, and, in certain areas, almost nonexistent. Here, we provide a brief overview of relevant research on bipolar disorders in the Arab world, point out major gaps in the literature, and offer some suggestions for future research.

\section{Literature review: bipolar patients in the Arab world}

Research on mental health in the Arab world has been lagging behind, with a recent review indicating that, over the last four decades, mental health publications in the Arab world represented less than $1 \%$ of the total medical publications from this region. ${ }^{1}$ Furthermore, many Arab journals are not listed in major biomedical search engines, such as PubMed and PsycInfo, and therefore a literature search on a specific topic can be challenging. For instance, a PubMed search using the keywords "bipolar disorder" and "Arab" revealed only 25 publications, several of which were irrelevant. A search using PsycInfo revealed similar results. We therefore used several other strategies in searching the literature, including a search by Arab country (e.g., Egypt, Lebanon, Tunisia, and Oman) and using specific search engines for Arab countries, such as Arabpsynet and the Institute for Development, Research, Advocacy, and Applied Care (IDRAAC). We also examined the 
references in key publications. The resulting summary of the identified relevant literature is categorized into three domains: epidemiology/clinical manifestations, etiology/biological mechanisms, and treatment/service utilization.

\section{Epidemiology and clinical characteristics}

A large part of the published literature in the Arab world consists of epidemiological surveys, such as that of mental disorders in an adult population attending a primary healthcare setting in Qatar. ${ }^{2}$ This prospective cross-sectional survey of 1660 respondents involved a face-to-face interview using a "designed diagnostic screening questionnaire" consisting of 79 questions on symptoms and signs of common mental health disorders. Following completion of the self-administered questionnaire, a provisional diagnosis was made, which was later confirmed by a psychiatrist using Diagnostic and Statistical Manual of Mental Disorders IV (DSM-IV) criteria. The results indicated that the prevalence of bipolar disorder among the Qatari population was $4.3 \%$, which, interestingly, was higher among female subjects $(5.3 \%)$ compared to males $(3.1 \%)$. In a prospective study of all psychiatric admissions to a general hospital in an Eastern province of Saudi Arabia between 1988 and 1998, ${ }^{3}$ a total of 1336 patients had 2217 admissions during that period; $19.5 \%$ were diagnosed with schizophrenia and $15.2 \%$ suffered from bipolar disorder. The diagnoses were made using "a fully-structured 45-item questionnaire specifically designed for data collection." Between 1998 and 1993, the diagnosis followed the DSM-IIIR criteria, and in 1993, the criteria shifted to the International Classification of Diseases 10 (ICD-10).

A more recent study estimated the prevalence of mental disorders among 1682 school-aged Omani adolescents and youth ${ }^{4}$ with face-to-face interviews using the Composite International Diagnostic Interview (CIDI): $:^{5} 13.9 \%$ had at least one DSM-IV diagnosis. The prevalence was $3 \%$ for major depressive disorder, $1 \%$ for bipolar disorder, and the highest was $9 \%$ for any anxiety disorder, including phobias. There were also data on mental health in specific countries, such as Lebanon, from large multinational surveys. The World Mental Health Survey Initiative, for instance, provides the lifetime prevalence of different mood disorders in Lebanon as follows: major depression (10.9\%), bipolar I disorder $(0.4 \%)$, bipolar II disorder $(0.5 \%)$, bipolar spectrum disorder (2.4\%), and subthreshold bipolar disorder $(1.5 \%){ }^{6}$ The data from Lebanon emerged from a population-based survey using the World Health Organization (WHO) CIDI version 3.0 , which was given to 2857 household members and had diagnostic criteria that followed the DSMIV. Criteria for subthreshold hypomania included at least one symptom of mania with failure to meet the full diagnostic criteria for hypomania.

Furthermore, several publications addressed the unipolar/bipolar dichotomy. For instance, Okasha et al., who estimated the frequency of bipolar disorder among patients with a major depressive episode in Egypt, found that 188 out of 302 (62\%) major depressive disorder patients could be diagnosed as bipolar using the Hypomania Checklist 32 (HCL-32) questionnaire. ${ }^{8}$ This compares with 42 out of $306(14 \%)$ when the DSM-IV is used and 144 out of $302(47 \%)$ when using other, less specific definitions of bipolarity. Underreporting of bipolar disorder was also found by a recent WHO multinational study ${ }^{9}$ of data from eight countries that participated in the World Mental Health Survey ( $n=47,552)$, including Lebanon $(n=2857)$. The results showed that many of the patients who were screened out for bipolarity on the basis of the CIDI could in fact be bipolar. The issue of bipolarity was further investigated by researchers in Tunisia who found that the polarity of the first episode in bipolar disorder could predict the course of the illness. In a retrospective study of 38 patients diagnosed with bipolar disorder in accordance with DSM-IV-TR, a first episode of mania or hypomania was associated with more frequent manic episodes and substance use. A first episode of depression, on the other hand, was predictive of a more chronic course, characterized by more episodes of depression with suicidal ideation and hypomania. ${ }^{10}$ Moreover, Douki et al. compared two samples of bipolar patients (40 subjects each) in two distinct geographical locations: Tunisia and France. ${ }^{11}$ All patients met DSM-IV criteria for bipolar disorder. The French patients were all from consecutive admissions to Centre Hospitalier Le Vinatier in Lyon, France, and the Tunisian patients were selected by matching the French patients on the basis of gender and duration of illness. The main difference between the two groups was the type of first episode: mania in three-quarters of cases in Tunisia and 
depression in three-quarters of cases in France. The same figures applied to the recurrences: more mania in Tunisia and more depression in France. Unipolar mania, although rarely mentioned in the Western literature, was three times more common in Tunisia than in France, according to these investigators. In a study by Dakhlaoui et al. ${ }^{12}$ that followed 72 DSM-IV bipolar patients who were first hospitalized at the Razi Hospital in Tunis between 1997 and 2001, recurrent mania and no episodes of depression was called unipolar mania, and the rest of the cases were called bipolar I. The authors noted that compared to patients with bipolar I, those with unipolar mania had an earlier age of onset (23 years vs. 27.6 years). They were also less likely to use substances and experienced less frequent affective episodes.

A number of articles addressed the issue of comorbidity of bipolar disorders. A retrospective comorbidity study from Lebanon ${ }^{13}$ assessed all patients hospitalized at the Psychiatric Unit of the St. George Hospital between 1979 and 1992. In 1643 charts available for review, 222 subjects (13.5\%) met DSM-IIIR criteria for substance abuse. Of these, 144 patients $(64.9 \%)$ were found to have concomitant psychiatric diagnoses. The mean age of onset of substance abuse was $25.9 \pm 10.5$ years and the mean duration of abuse was $8.1 \pm 8.5$ years. Among patients with dual diagnoses, $42 \%$ of cocaine abusers suffered from some form of bipolar disorder, whereas $45 \%$ of cannabis abusers also suffered from schizophrenia. Heroin users, however, were more likely to suffer a personality disorder, specifically antisocial personality. Furthermore, Erfan and Amer ${ }^{14}$ assessed the prevalence of nicotine dependence among consecutive admissions to a private hospital in Egypt. They limited their study to patients with schizophrenia $(n=70)$ and bipolar disorder $(n=46)$. They found that $58.6 \%$ of the schizophrenic patients were smokers $(60 \%$ were dependent) compared to $32.6 \%$ of the bipolar patients $(38.5 \%$ were dependent). With respect to medical comorbidity, Ezzaher et al. ${ }^{15}$ reported that Tunisian bipolar patients were more likely to be obese than controls (30\% vs. $12 \%$ ) and also had higher cholesterol and low-density lipoprotein (LDL) values and lower high-density lipoprotein (HDL) values than controls. The specific role of medications, such as valproic acid, in the treatment of such comorbid conditions was not specified.

\section{Etiology/pathophysiology}

Compared to the literature addressing epidemiology and/or clinical manifestations of bipolar disorders in the Arab world, the literature dealing with etiology and/or pathophysiology is even more difficult to find. There were several studies that assessed the effects of consanguinity on mental health. As marriage between cousins is a common practice and culturally accepted in this part of the world, ${ }^{16}$ it is not surprising that researchers assessed the impact of consanguinity on different aspects of mental health. For example, Saadat ${ }^{17}$ investigated whether parental consanguineous marriages could affect the age of onset of bipolar disorder in a subject population of 195 bipolar disorder patients. All patients were diagnosed in accordance with DSM-IV criteria. The study found that the age of onset was earlier for the offspring of consanguineous marriages compared to unrelated controls, but only for an age of onset above 25 years. In two independent, systematic studies on the rate of consanguinity among patients with bipolar I disorder in Egypt, Mansour et al. ${ }^{18}$ compared the rate of consanguinity among Egyptian bipolar patients and appropriate controls. The first study was a case-control study of 93 bipolar patients diagnosed in accordance with DSM-IV criteria and 90 screened controls. Consanguinity was determined by self-report and confirmed by DNA analysis. There were 34 parental consanguineous marriages for the 93 bipolar patients $(36.5 \%)$ and only 16 parental consanguineous marriages for the 90 control subjects $(17.7 \%)$, a difference that was statistically significant $(P<0.005)$. The second study was a community-based survey of psychiatric morbidity that used the Mini International Neuropsychiatric Interview (Arabic version). ${ }^{19}$ Out of 1584 subjects screened, 35 subjects were identified as bipolar patients and 150 randomly selected, unaffected subjects were identified as controls. The rate of parental consanguinity was estimated by selfreport only. In this study, the corresponding figures for parental consanguinity were 16 of $35(45.7 \%)$ for the bipolar patients and 23 of $150(15.1 \%)$ for the controls $(P<0.0001)$. In a later study, the authors examined the possibility that the association between increased risk of bipolar disorder among consanguineous families could be mediated by telomere length. ${ }^{20}$ They measured telomere length in 108 bipolar patients and 114 matched 
controls and found no differences in telomere length between the groups and no relation to consanguinity.

There have also been reports of associations between specific genetic polymorphisms and bipolar I disorder in various Arab populations. In one study, 79 patient-parents triads of bipolar patients were analyzed in a Palestinian Arab population. ${ }^{21}$ Using the transmission disequilibrium test, the authors stated that the results are "consistent with previous reports of linkage between microsatellite marker D22S278 and bipolar disorder." Furthermore, in a study of 109 bipolar patients meeting DSM-IV criteria and 110 controls in a Tunisian population, a genotype analysis that was carried out by multiplex polymerase chain reaction (PCR) showed an association between the L55M and Q192R polymorphisms of paraoxonase 1 (PON1) and bipolar I disorder. ${ }^{22}$ A recent study from Egypt focused on the association between the methylenetetrahydrofolate reductase (MTHFR) polymorphism and schizophrenia and bipolar disorders ${ }^{23}$ among 103 schizophrenic patients, 134 bipolar patients, and 149 controls, with diagnoses made in accordance with DSM-IV-TR criteria and using the MINI. The authors found a higher prevalence of the MTHFR C677T polymorphism in bipolar patients and schizophrenia than in the healthy controls. Another recent study investigated the association of two genes encoding the enzyme myo-inositol monophosphatase 1 (IMPA1) and myo-inositol monophosphatase 2 (IMPA2) with bipolar disorder. The sample consisted of 74 Palestinian proband-parent trios, and all affected offspring $(n=92)$ met DSM-IV criteria for bipolar disorders, using the SCID interview. The authors reported a significant association between IMPA2 and bipolar disorder in the trio sample of Palestinian Arabs. ${ }^{24}$ These studies, however, remain isolated and suffer from relatively small numbers of subjects. It is often unclear whether the studies were sufficiently powered for statistical analyses. With a database so small, we did not find any systematic reviews or meta-analyses.

In addition to the genetic studies in bipolar patients, there are biological reports on neurochemical, neuroendocrine, or immune parameters. One study from Morocco explored the effects of fasting during Ramadan on the course of illness in 20 lithium-treated patients with stable bipolar disorder diagnosed in accordance with ICD-10 criteria. ${ }^{25}$ The authors reported a relapse rate of $45 \%$ during Ramadan in spite of adequate plasma lithium concentrations. They attribute the relapse to a change in "social rhythm." Another study from Tunisia investigated the presence of hyperhomocysteinemia in 92 DSM-IV-diagnosed bipolar disorder patients and 170 controls, and attempted to relate hyperhomocysteinemia to the 5,10-MTHFR C677T polymorphism. ${ }^{26}$ They found a significant increase in blood levels of homocysteine in bipolar disorder patients, but this change was independent of the C677T polymorphism. They also found a significant decrease in the blood levels of folic acid. The hyperhomocysteinemia, they noted, was associated with the duration of the depressive episode, whereas the hypofolatemia was associated with lithium and/or carbamazepine use.

In a different study also from Tunisia, the authors assessed the variations in the activity of paraoxanase, an enzyme associated with the synthesis of lipoprotein particles, and the lipid profile of 66 bipolar disorder patients, diagnosed in accordance with DSM-IV criteria, and 64 matched controls. Compared to the controls, the patients had significantly lowered paraoxanase activity and ApoA1 levels and significantly higher total cholesterol, low-density protein cholesterol (c-LDL) and lipoprotein (a) (Lp (a)). ${ }^{15}$ The relation to the risk of cardiovascular disease in these patients was not assessed. The prevalence of obesity and overweight in Tunisian bipolar patients was evaluated by the same group, ${ }^{27}$ who studied 130 DSM-IV-diagnosed bipolar patients and 130 matched controls. They found the prevalence of obesity to be much higher in bipolar patients (30.1\%) compared to the controls $(12.3 \%)$. The observed increase in body mass index in the bipolar patients was not related to gender or tobacco or alcohol use, but could be associated with the use of valproic acid. Furthermore, the presence of immunological perturbations in bipolar disorder patients was assessed by a group of Egyptian investigators $^{28}$ who compared the percentages of lymphocytes, active T-lymphocytes, and natural killer (NK) cells in 20 manic patients and 20 healthy controls. They found a significant decrease in the percentage of lymphocytes and NK cells in the manic group compared to controls; however, the number of activated T-lymphocytes was not changed. These data from the Middle East add to 
the growing world literature suggesting immune and/or inflammatory changes in patients with bipolar disorders.

\section{Service utilization/treatment}

Not surprisingly, quality research in the areas of service utilization and treatment in the Arab world is also scarce. Several reports address service utilization in general as it relates to specific countries or regions, reasons why patients are frequently noncompliant with their medications, and patterns of psychiatric hospitalizations. For example, Al Riyami et al. ${ }^{29}$ conducted face-to-face interviews using the CIDI with 1836 Omani school-aged children and young adults between the ages of 14 and 23 years; only $13.2 \%$ of patients with any DSM-IV mood disorder made treatment contact for their disorder during the first year. For patients with bipolar disorder, the median delay in initial treatment contact was 2 years, and male patients were significantly less likely to make treatment contact than their female counterparts. In addition, Bener et al. ${ }^{30}$ studied reasons for medication noncompliance among 564 hospitalized psychiatric patients who were followedup in the outpatient clinic of a psychiatry department in Doha, Qatar. Three-hundred twenty-eight patients (58\%) were considered medication compliant, and the remaining 236 (41.8\%) were considered noncompliant; $12.7 \%$ of bipolar disorder patients were noncompliant compared to a noncompliance rate of $28.4 \%$ among schizophrenic patients. Reasons for noncompliance included poor social supervision $(40 \%)$, irregular clinic attendance $(55.5 \%)$, and a lack of education about medication (58.1\%). The authors strongly encouraged better health education of patients at the community level.

Most reports that focus on treatment of bipolar disorder in the Arab world tend to be general and often consist of a simple description of the product and its clinical usefulness as seen by the authors. There are also occasional critical reviews of the topic and informed opinions, ${ }^{31}$ as well as articles written in favor of specific medications. It is therefore not surprising that lithium gets the most attention in this regard, as lithium is the treatment standard for bipolar disorders. In a critical review by Abou Saleh on the prediction of lithium response in patients with affective disorders, ${ }^{32}$ it was concluded that accuracy of the diagnosis, personality factors, and early response remain the best predictors. ${ }^{33}$ Other articles address issues such as lithium clearance in an Egyptian population ${ }^{34}$ and a new and accurate method for predicting daily lithium dosage requirements. ${ }^{35}$ There were also isolated reports on other medications, such as the efficacy and safety of risperidone in psychotic patients ${ }^{36}$ and the use of clozapine as a mood stabilizer in chronic and treatment-resistant bipolar disorder patients. ${ }^{37} \mathrm{We}$ were surprised by the near absence of results from clinical trials taking place in the Arab world. As an example, a search for "clinical trials" in the search engine IDRAAC returned nine articles. On closer examination, five of the studies were not conducted in the Arab world, and the four others were review articles written by Arab authors. Not a single paper reported on results of randomized controlled trials conducted in the Arab World on psychotropic medications.

\section{Critical assessment}

This review revealed to us major gaps in the Arab psychiatry literature in general and in the literature on bipolar disorders specifically. One major problem in the literature search was the absence of relevant search engines for the Arabic medical literature or in Arabic. Most psychiatric journals published in the Arab world are not included in PubMed or PsycInfo. There are regional search engines, such as Arabpsynet and IDRAAC, but more work needs to be done to improve their effectiveness in specific literature searches.

The methodology sections in many manuscripts published in the Arab psychiatry literature is superficial and/or incomplete. Diagnostic criteria are often mentioned but without the needed information on the specific diagnostic tools used, the individuals making the diagnoses, or the training of the personnel using diagnostic interviews. Furthermore, although many of these structured diagnostic interviews are available in Arabic, information about adaptation and validation in the local culture is often lacking. The statistical analysis is rarely adequately discussed, and very few reports address the issue of statistical power and whether sample sizes are adequate enough to answer the research question; advanced statistical analyses are rare. In addition, the research question and the methods used to address it are not always in harmony.

As to the research topics, most reported studies are observational and/or descriptive in nature. 
Limited epidemiological and clinical observations, including case reports, are common; however, we have not found large systematic analyses or metaanalyses. Biological reports, including genetic studies, are frequently underpowered, and randomized controlled drug trials are almost nonexistent. The best studies with respect to methodologies and standards seem to be those that are part of a regional or multinational investigation (e.g., WHO studies), in which an individual Arab country or countries take part. ${ }^{6,7,9}$

\section{Suggestions for the future}

In view of the gaps addressed above, it seems reasonable to continue and intensify efforts to translate studies into Arabic and adapt and validate representative and widely used diagnostic interviews and psychiatric rating scales that should be used routinely in all Arabic clinical reports; IDRAAC in Lebanon has made significant efforts in this regard. ${ }^{38}$ Access to these tools should also be made easy for researchers to incorporate them in their daily work.

Another priority should be serious epidemiological work using the DSM-V ${ }^{39}$ and/or its global counterpart ICD-11. ${ }^{40}$ The DSM-V has recently been adopted by the American Psychiatric Association after many years of hard work to capture the latest knowledge about different psychiatric disorders. The DSM-V also recognizes cultural variations for many disorders and efforts to illustrate this section with Arabic examples should be undertaken. Although the ICD-11 is still in the final stages of its development, it has incorporated field studies in different regions of the world to assess its use among the local population. A group of clinicians representing the Arab world has been actively engaged in this endeavor. ${ }^{41}$ The group is tasked with making suggestions and recommendations on the basis of field studies conducted in various regions of the Arab world. This area of research is therefore ready for serious epidemiological work for the DSM-V and/or the ICD-11 to be undertaken in Arab countries.

It is also very important for Arab investigators to collaborate on large projects, such as the genomics of affective disorders. Individual studies should have a minimum set of requirements, such as standardized and validated diagnostic criteria, ${ }^{38}$ state-of-the-art sequencing procedures, and a large pool of patients and controls. Most of the current genome-wide association studies in psychiatric disorders have been done on Western European populations; ${ }^{42}$ there are no sufficiently large samples of Arab populations available to study in order to test such associations. It is to be anticipated that there will be a combination of unique and overlapping genetic variations among Arab bipolar disorder patients relative to Western populations, but this remains unknown at the present time. Large and systematic collaborative efforts will be needed to establish samples that are ascertained and assessed using common diagnostic instruments and similar methodological approaches. Similar approaches need to be followed for randomized controlled trials in Arab countries assessing the efficacy and safety of newer antidepressants and mood stabilizers. Only then will research on bipolar disorders in the Arab world be in a position to advance science and contribute to a better understanding of this disorder.

\section{Conclusion}

In many ways, bipolar disorder patients in the Arab world are similar to bipolar patients in other regions of the world. For example, the reported prevalence is in the range of $1-5 \%$. The disorder can be found in all countries, affecting both genders about equally, and causing a great deal of distress and functional impairment. It is when one looks more closely that differences become more apparent. Specifically, bipolar disorder patients in the Arab world suffer from more frequent manic episodes, and there is a higher rate of consanguinity among parents of bipolar patients than among parents of controls. Psychiatric comorbidity may not be as common, particularly in relation to alcoholism and substance abuse. Medical comorbidity, however, is frequent, especially with obesity and the metabolic syndrome.

In summary, bipolar disorder in the Arab world has features that directly overlap with those in other regions, which provides the basis for comparison of risk, susceptibility, and protective factors. However, there are also unique features, such as consanguinity and higher rates of mania, that provide the opportunity for a more focused approach that would allow Arab researchers to make specific contributions to the field using data sets with more standardized methodologies and larger numbers of subjects.

\section{Conflicts of interest}

The authors declare no conflicts of interest. 


\section{References}

1. Jaalouk, D. et al. 2012. Mental health research in the Arab world. Soc. Psychiatry Psychiatr. Epidemiol. 47: 1727-1731.

2. Ghuloum, S., A. Bener \& M.T. Abou-Saleh. 2011. Prevalence of mental disorders in adult population attending primary health care setting in Qatari population. J. Pakistan Med. Assoc. 61: 216-21.

3. AbuMadini, M.S. \& S.I. Rahim. 2002. Psychiatric admission in a general hospital: patients profile and patterns of service utilization over a decade. Saudi Med. J. 23: 44-50.

4. Jaju, S.1., S. Al-Adawi, H. Al-Kharusi, et al. 2009. Prevalence and age-of-onset distributions of DSM IV mental disorders and their severity among school going Omani adolescents and youths: WMH-CIDI findings. Child Adolesc. Psychiatry Ment. Health 3: 29.

5. Kessler, R.C. \& T.B. Ustun 2004. The World Mental Health (WMH) Survey Initiative version of the World Health Organization (WHO) Composite International Diagnostic Interview (CIDI). Int. J. Methods Psychiatr. Res. 13: 93-121.

6. Merikangas, K.R. et al. 2011. Prevalence and correlates of bipolar spectrum disorder in the world mental health survey initiative. Arch. Gen. Psychiat. 68: 241-251.

7. Okasha, T. et al. 2013. Screening for bipolar disorder among patients undergoing a major depressive episode: report from the BRIDGE study in Egypt. J. Affect Disord. 147: 217-224.

8. Angst, J., Adolfsson, R., Benazzi, F., et al. 2005. The HCL-32: towards a self-assessment tool for hypomanic symptoms in outpatients. J. Affect Disord. 88: 217-233.

9. Karam, E.G., N. Sampson, L. Itani, et al. 2014. Under-reporting bipolar disorder in large-scale epidemiologic studies. J. Affect Disord. 159: 147-154.

10. Bram, N. et al. 2012. Clinical and evolutionary characteristics of bipolar disorder according to the polarity of the first episode. Tunis Med. 90: 380-386.

11. Douki, S. et al. 2012. Cross-cultural aspects of bipolar disorder: results of a comparative study between French and Tunisian patients. Encéphale. 38: 194-200.

12. Dakhlaoui, O. et al. 2008. Clinical particularism of bipolar disorder: unipolar mania, about a patient's study in Tunisia. Encéphale. 34: 337-342.

13. Karam, E.G. et al. 2002. Comorbidity of substance abuse and other psychiatric disorders in acute general psychiatric admissions: a study from Lebanon. Comprehensive Psychiat. 43: 463-468.

14. Erfan, S. \& D. Amer. 2007. Nicotine dependence in schizophrenic and bipolar disorder patients: a comparative study. Egypt J. Psychiat. 26: 13-19.

15. Ezzaher, A. et al. 2010. Lower paraoxonase 1 activity in Tunisian bipolar 1 patients. Ann. Gen. Psychiat. 9: 36-42.

16. Harkness, G., R. Khaled. Modern traditionalism. 2014: consanguineous marriage in Qatar. J. Marriage Family 76: 587-603.

17. Saadat, M. 2012. Influence of parental consanguineous marriages on age at onset of bipolar disorder. Psychiat. Res. 198: 327-328.

18. Mansour, H. et al. 2009. Consanguinity associated with increased risk for bipolar I disorder in Egypt. Am. J. Med. Genet. Part B: Neuropsychiat. Genet. 150: 879-885.
19. Sheehan, D.V. et al. 1998. The Mini International Neuropsychiatric Interview (MINI): the development and validation of a structured diagnostic psychiatric interview for DSM-IV and ICD-10. J. Clin. Psychiat. 59(Suppl. 20): 22-33

20. Mansour, H. et al. 2011. Does telomere length mediate associations between inbreeding and increased risk for bipolar I disorder and schizophrenia? Psychiat. Res. 188: 129-132.

21. Mujaheed, M. et al. 2000. Evidence for linkage by transmission disequilibrium test analysis of a chromosome 22 microsatellite marker D22S278 and bipolar disorder in a Palestinian Arab population. Am. J. Med. Genet. 96: 836838.

22. Ezzaher, A. et al. 2012. Association between bipolar I disorder and the L55M and Q192R polymorphisms of the paraoxonase 1 (PON1) gene. J. Affect Disord. 139: 12-17.

23. El-Hadidy, M.A.1., H.M. Abdeen, S.M. Abd El-Aziz, et al. 2013. C677T Methylenetetrahydrofolate reductase gene polymorphism in schizophrenia and bipolar disorder. Psychiatr. Genet. DOI:10.1097/YPG.0000000000000020.

24. Sjøholt, G.1., R.P. Ebstein, R.T. Lie, et al. 2004: Examination of IMPA1 and IMPA2 genes in manic-depressive patients: association between IMPA2 promoter polymorphisms and bipolar disorder. Mol. Psychiat. 9: 621-629.

25. Kadri, N. et al. 2000. Relapses in bipolar patients: changes in social rhythm? Intl. J. Neuropsychopharm. 3: 45-49.

26. Ezzaher, A. et al. 2011. Hyperhomocysteinemia in Tunisian bipolar I patients. Psychiat. Clin. Neurosci. 65: 664-671.

27. Ezzaher A. et al. 2009. Obesity and dyslipidemia in Tunisian bipolar subjects. Ann. Biol. Clin. 68: 277-284.

28. Abeer, A.E. \& H.A. Ramy. 2006. Immunological changes in patients with mania: changes in cell-mediated immunity in a sample from Egyptian patients. Egypt J. Immunol. 13: 79-85.

29. Al Riyami, A.A. et al. 2009. Health services utilization by school going Omani adolescents and youths with DSM IV mental disorders and barriers to service use. Intl. J. Ment. Health Syst. 25: 11-22.

30. Bener, A. et al. 2013. A study of reasons of non-compliance of psychiatric treatment and patients' attitudes towards illness and treatment in Qatar. Issues Ment. Health Nurs. 34: 273280.

31. Okasha, A. 2004. State of the art in the management of bipolar disorder. Arab J. Psychiat. 15: 1-7.

32. Abou-Saleh, M.T. 2000. Prediction of response to lithium in affective disorders. Arab J. Psychiat. 11: 88-94.

33. Abou-Saleh, M.T. \& A.J. Coppen. 1990. Predictors of longterm outcome of mood disorder on prophylactic lithium. Lithium Clinic Epsom 1: 27-35.

34. ElDesoky, E.S. et al. 2008. Estimation of lithium clearance from routine clinical data in Egyptian bipolar patients: a population pharmacokinetic approach. Intl. J. Clin. Pharmacol. Therap. 46: 617-626.

35. Abou-Auda, H.S. et al. 2008. A new accurate method for predicting lithium clearance and daily dosage requirements in adult psychiatric patients. Bipolar Disord. 10: 369-376. 
36. Daradkeh, T.L. et al. 1996. Efficacy and safety of resperidone in psychotic patients: an open study. J. Intl. Med. Res. 24: 291-295.

37. Alhamad, A.M. 2003. Clozapine: a mood stabilizer in chronic resistant bipolar affective disorder. Arab J. Psychiat. 14: 88-93.

38. Karam EG, Kronfol Z, Salamoun M, et al. 2014. The arabic diagnostic interview for genetic studies: aDIGS: Psychometric properties. Arab J. Psychiat. 25: 18-23.

39. American Psychiatric Association. 2013. Diagnostic and Statistical Manual of Mental Disorders. 5th ed. Washington, DC.
40. Maj, M., \& G.M. Reed. 2012. The development of the ICD-11 classification of mood and anxiety disorders. World Psychiat. 11(Suppl. 1): 3-5.

41. Khoury, B., N. Loza \& G.M. Reed. 2011. Arab specificities, arab voice and global connectedness: the development of WHO's new international classification of mental disorders (ICD11). Arab J. Psychiat. 22: 95-99.

42. Smoller, J.W., N. Craddock, K. Kendler, et al. 2013. Identification of risk loci with shared effects on five major psychiatric disorders: a genome-wide analysis. Lancet 381: 1371-1379. 